\title{
Children's subjective well-being in Africa: A comparative analysis across three countries
}

Shazly Savahl, Habib Tiliouine, Ferran Casas, Sabirah Adams, Yehualashet Mekonen, Negussie Dejene, Elizabeth Benninger and Heidi Witten

\begin{abstract}
Recent trends in child well-being research have shown a substantial advancement in studies investigating children's subjective well-being (SWB). This advancement has raised questions concerning the measurement of SWB and the extent to which various measures can be compared across countries and diverse cultures. With a dearth of empirical data on cross-cultural comparisons, the validation of existing measures and cross-cultural comparisons and adaptations, have been identified as a critical course of action. The current study contributes to this process - it aims to report on children's SWB in three African countries (Algeria, Ethiopia, and South Africa), using two multiple item measures of SWB (the context-free Students' Life Satisfaction Scale and the domain- specific Personal WellBeing Index-School Children). Within this process the study further aims to test the structural validity of these measures and to ascertain its cross-country comparability. Data from the second wave of the Children's Worlds survey were used; and includes a randomly selected sample of 3394 children between the ages of 11-12 from Algeria (Provinces of El Bayedh, Oran, and Tlemcen), Ethiopia and South Africa (Western Cape Province). Located within the goodness of fit theoretical framework, confirmatory factor analysis and Structural Equation Modelling were used to test the overall fit structure, while multi-group confirmatory factor analysis was used to test measurement invariance. The results show appropriate fit structure for the individual models, with metric and scalar factor invariance tenable across the three countries for the Students' Life Satisfaction Scale and partial scalar invariance obtained for four items on the Personal Well-Being Index-School Children. The Algerian sample scored significantly higher than Ethiopia and South Africa on both SWB measures. Appropriate fit structure was obtained for the combined model and for the structural model, indicating adequate convergent validity with the single item Overall Life Satisfaction. Metric and partial scalar invariance was tenable for the structural model, suggesting cross-country comparability for correlations, regressions and means. The overall findings suggest that the two measures are appropriate for use with children from the three countries and that meaningful comparisons can be made between the three countries.
\end{abstract}




\section{Introduction}

Seminal work on the multi-dimensional measurement of child well- being can be located in two research movements in the mid 1970's, namely the social indicators and quality of life movements, founded by Campbell, Converse, and Rodgers (1976) and Andrews and Withey (1976) (in North America), and Allardt (1976, in Scandinavia) (see Camfield, Streuli, \& Woodhead, 2010). Early efforts in the measurement of children's well-being emphasised the development of all-inclusive indices which were constructed from established indicators evident in the work by the aforementioned scholars. It was in the late 2oth century that measures and indices focusing on children were developed, ad- dressing the critique of early indices which did not consider children's subjective perceptions (Watts \& Hernandez, 1982). The children's right's movement was also influential in the establishment of the child in- dicator's movement, especially in recognising the dignity and best in- terests of the child (Casas, 2016; Sandin, 2014). Since its genesis, the field of child indicators has focused on providing a contextual and multicultural understanding of child well-being. Recent changes in the child indicators movement has embodied a shift from: child survival and well-becoming to child well-being, a single discipline to a multi- disciplinary focus, national geographic units to a variety of geo- graphical units, a focus on negative to positive outcomes. This shift in focus has also advanced a children's rights perspective, a focus on both children's and adult's perspectives, and a close connection to social policy, and a focus on children's subjective well-being (SWB) (Ben- Arieh, 2010). Camfield et al. (2010) note that less than one percent of early indices on child well-being were employed in developing coun- tries. Work by Tiliouine (2012; Casas, Tiliouine, \& Figuer, 2014; Tiliouine, Cummins, \& Davern, 2006) focusing on adults and children's well-being in Algeria, and recent research by Savahl et al. (2015) and Savahl, Casas, and Adams (2016) in South Africa has contributed to this dearth in research from developing contexts, particularly from Africa. The current study hopes to make further contributions in this regard. The overarching aim of the study is to provide a general overview children's SWB in three African countries (Algeria, Ethiopia, and South Africa) using two measures of SWB. More specifically the study aims to ascertain the extent to which the two SWB measures allow for cross- cultural comparison.

The changing field of child well-being has also led to an increase in research which incorporates an interest in communities and neigh- bourhoods as the context of children's well-being and a growing emergence of reports related to children's well-being from nonwestern and non- English speaking countries (Ben-Arieh, 2010). Furthermore, children are increasingly being recognised within scientific research as competent informers regarding aspect of their own lives (Casas, 2016). In addition to exploring children's subjective understandings and ex- periences of their well-being, research should consider the particular social and historical contexts which influence children's evaluations and outcomes (Camfield et al., 2010). More so, as research on SWB provides valuable information to policy makers, it has the potential to contribute to better understandings of the causes and correlates of SWB within a particular population. Along with advocacy for children's wellbeing has been the promotion of children's rights as enshrined in broad in- ternational policy frameworks such as the United Nations Convention on the Rights of the Child 
(UNCRC), and the African Charter on the Rights and Welfare of the Child (ACRWC) (www.unicef.org/esaro/ children_youth_5930.html). These policy frameworks advance the col- lection of reliable data on matters affecting the child. For example, the ACRWC highlights the rights that African countries should ensure for their children, and is the primary instrument of the African human rights system for promoting, and protecting child rights. (www.unicef. org/esaro/children_youth_5930.html).

Africa has seen great achievements over the past ten years in rela- tion to the status of children's well-being. The most notable of these achievements is the increased survival rate, including a huge reduction of infant mortality, along with fewer children and parents dying of conflict-related causes (ACPF, 2013). Additionally, the access to water, sanitation, and education has increased significantly. The countries which have put in place national laws and policies to protect children from violence and maltreatment and which have allocated adequate budgets for children's sectors and well-being outcomes, have received the highest child-friendliness ratings (ACPF, 2013). On the contrary, the least child-friendly countries are those which have failed to implement appropriate policy frameworks to protect children from abuse, and which have not made significant efforts towards the improvement of children's access to basic services. This emphasises a country's child- friendliness to be closely related to their political and budgetary com- mitment towards children's policies and services, rather than its wealth or level of development. While in many ways the improvements in children's well-being in Africa seems to be improving, there remains a number of barriers which prevent many of Africa's children from ac- cessing the necessary resources for an adequate quality of life. This includes persistently high levels of malnutrition throughout Africa, ongoing conflict within many countries, inequitable distribution of ser- vices, education inequality for girls, and inadequate commitment to children's participation around issues affecting their lives.

In terms of national objective indicators of the three countries under investigation, Algeria has the lowest under-5 and infant mortality rates (Under-5 mortality: Algeria, 25 per 1000; Ethiopia, 64 per 1000; and South Africa, 43 per 1000; Infant mortality [per 1000 live births]: Algeria, 22; Ethiopia, 64; South Africa, 33 [World Bank, 2016]). In terms of gross domestic product (GDP), South Africa (350.63) fares better than Algeria (210.18), while Ethiopia (47.53) is substantially lower. Ethiopia also fares less satisfactorily in terms of the percentage of children underweight (29.2\%), and children who are stunted (44\%). Of the three countries, South Africa remains the African country with the highest level of inequality, with a gini index of 63 , and globally ranked fourth in terms of inequality (World Bank, 2016, http://databank. worldbank.org/data/reports.aspx?source=worlddevelopment- indicators\#).

The African Child Policy Forum (ACPF) (2013) has identified the five priority areas for action to improve the life satisfaction of children:

1. Strengthening systems and capacities to enhance accountability to children

2. Further improving the survival of children and their access to basic needs and services 
3. Increasing budgetary allocations to programmes benefiting children, and enhancing commitment to address growing inequality

4. Providing full legal protection for children and strengthening en- forcement

5. Putting in place mechanisms to ensure children's participation in decisions that affect them (p. XVIII).

In order to successfully monitor, implement, and track the progress related to the improvement of children's rights and overall well-being, African countries must invest in regular collection of information re- garding the status of children's rights and perceptions and evaluations of their well-being (ACPF, 2013). The important concern then arises of how to accurately measure children's SWB.

Previously, the only psychometric scale assessing well-being was the single-item scale the 'Cantril Ladder' (Casas, Bălțătescu, Bertran, Gonzalez, \& Hatos, 2013; Casas, Bello, Gonzalez, \& Aligué, 2013; Casas et. al, 2014). More recent evidence suggests that the use of multi-item measures of SWB are more stable than single items (Casas, Bălțătescu, et al. 2013; Casas, Bello, et al., 2013; Casas et al., 2014), reduces the risk of measurement error, and increases the reliability of the measure. Amongst the most widely used scales are those that measure global life satisfaction (context-free) (Student's Life Satisfaction Scale [SLSS], Huebner, 1991) and those measuring multiple dimensions (Personal Well-Being IndexSchool Children [PWI-SC], Cummins \& Lau, 2005; the Multidimensional Student Life Satisfaction Scale [MSLSS], Huebner, 1994, and the Brief Multidimensional Student Life Satisfaction Scale [BMSLSS], Huebner, Seligson, Valois, \& Suldo, 2006). These scales have been found to present with sound structural validity amongst children and adolescents in developed countries such as Spain (Casas, Bălțătescu, et al. 2013; Casas, Bello, et al., 2013; Casas et al., 2014), Portugal (Marques, Pais-Ribeiro, \& Lopez, 2007), Germany (Weber, Ruch, \& Huebner, 2013), Australia (Tomyn, Norrish, \& Cummins, 2013), Turkey (Siyez \& Kaya, 2007), and China (Ye et al., 2014; Tian, Zhang, \& Huebner, 2015). Historically, less information was available with regard to the validation of SWB scales in less developed countries. However, recently there has been a number of research initiatives aimed at the adaptation and validation of SWB instruments in devel- oping contexts. For example a study by Savahl et al. (2016) conducted amongst a sample of 12- year old children in South Africa, found the SLSS and PWI-SC to be valid and reliable measures. Similarly studies conducted by Alfaro et al. (Alfaro, Guzman, Garcia et al., 2016; Alfaro, Guzman, Sirlopu et al., 2016) in Chile amongst a sample of children and adolescents found the SLSS and the PWISC to be valid and reliable measures. Furthermore, Sarriera, Bedin, and Abs (2013) tested the SLSS, PWI-SC, and BMSLSS amongst a sample of 9-13 year old children in Brazil, while Sarriera et al. (2014) tested the PWI-SC (including items on spirituality and religion) on a sample of Brazilian and Chilean adolescents. The authors found these instruments to be valid and reli- able for use amongst children and adolescents in Brazil and Chile. Fi- nally, a study by Siyez and Kaya (2007) amongst conducted with children and adolescents in Turkey found the BMSLSS to have appro- priate psychometric properties; while studies in Serbia by Jovanovic and Zuljevic (2013) and Iran by Habibi, Pooravari, Salehi, Fard, and Pooravari (2015) found the MSLSS to be a valid measure for use amongst adolescents. 
The importance of cross-cultural comparisons between developed and developing contexts has been advanced in contemporary literature. Casas and Rees (2015), using data from the Children's Worlds project highlight the significance of conducting cross-cultural and international comparative studies on children's SWB. They found that cross-national comparisons by correlations and regressions were tenable but that comparisons by means were not. Similarly, a cross-cultural comparative study including 23 countries using the BMSLSS (Abubakar et al., 2015) with a sample of adolescents and emerging adults found appropriate structural validity but cautioned against mean comparisons across cultures. Further crosscultural comparative studies between: Spanish and Algerian children (Casas et al., 2014), Spanish and Romanian adolescents (Casas, Bălțătescu, et al., 2013), Spanish, Chilean, and Brazilian adolescents (Casas et al., 2012), and Australian and Portu- guese adolescents (Tomyn, Tamir, Stokes, \& Dias, 2015), demonstrate appropriate structural validity and crosscultural comparability of a range of SWB instruments across diverse cultures. More recently, Casas (2016) has pointed to the importance of including multiple scales with items of different levels of abstraction when investigating children's SWB. The authors of these cross-cultural studies recommend further international and cross-cultural comparisons of SWB instruments across diverse contexts. This study hopes to make a contribution to the lit- erature in this regard.

\subsection{Aim of the study}

The overarching aim of the current study is to provide a general overview of children's SWB in three African countries (Algeria, Ethiopia, and South Africa) using two multiple item SWB measures. The study further aims to test the structural validity of the measures and to ascertain the extent to which they are comparable across the three African countries (Algeria, Ethiopia, and South Africa). The following objectives have been developed to guide the study:

a) To determine the subjective well-being of a sample of children from Algeria, Ethiopia, and South Africa

b) To determine the structural validity of two multiple- item SWB measures assessing context-free (SLSS) and domain-specific (PWI-SC) subjective well-being

c) To determine measurement invariance of the SLSS and the PWI-SC across Algeria, Ethiopia, and South Africa

\section{Method}

\subsection{Design}

The study uses data from Wave II of the Children's Worlds Project: International Survey of Children's Well-Being (ISCWeB), a collaborative international study which aims to explore children's SWB (see www. isciweb.org). This wave included 15 countries from across Africa, Asia, Europe, the Middle East, and South America. Amongst the African countries which participated in the study are Algeria (Provinces of El Bayedh, Oran, and Tlemcen), Ethiopia, and South Africa (Western Cape Province). The Children's Worlds Project employed a crosssectional design, with a focus on children aged 8, 10, and 12 years (Rees \& Main, 2015). 
Within the Children's Worlds Project each country aimed to include a representative sample across the three age cohorts of 8,10, and 12- year old. All countries in the project employed probability sampling methods. The target for the survey was to collect data with 1000 chil- dren in each age cohort. The current study uses data from the 12- year old dataset of Algeria, Ethiopia and South Africa and includes a total sample of 3394 children between the ages of 11-12 years. The sampling strategy followed in each of the three countries under consideration is described below.

2.2.1

Algeria

The sample from Algeria comprised learners from school year 3 (Primary School- 3PS), 5 (Primary School- 5PS), and 1st Middle School (1MS) in its Western region. An expert panel was employed to select three provinces from a total of 11 within the particular region, namely El Bayedh, Oran, and Tlemcen. From within these three regions, schools were randomly selected. Within each province (stratum), schools were selected proportionate to the number of pupils, with the aim to include eight schools from each stratum. Two classes per school were then se- lected, which did not bias any children. The questionnaire was trans- lated into Arabic, which is the language-medium of school teaching in Algeria. The final overall sample from Algeria included 3600 children between 8 and 12 years, with 1200 participants in each age cohort.

\subsection{2}

\section{Ethiopia}

The sampling procedure for the survey in Ethiopia included a three- stage cluster method. In stage one, 50 districts were selected randomly from across the country using a 'Probability Proportional to Size' (PPS) technique, where the 'size' component was relative to the number of children enrolled in primary schools in the particular districts. In stage two, two schools were randomly selected from each district, and in- cluded a total of 100 schools from 50 districts. In stage three, children from the three age cohorts of 8, 10, and 12-years were randomly se- lected. School registers were used as the sampling frame for selection of participants. Ten students from each of the three grades were selected, resulting in 30 children from each school, with an equal gender com- position across age cohorts. The questionnaire was translated into Amharic, using the backward translation method. The final sample in- cluded 3000 children, with 1000 children in each age cohort (Mekonen \& Dejene, 2015).

\subsubsection{South Africa}

The participants for the sample from South Africa were selected from eight Education Management District Councils (EMDC) of the Western Cape Province, which included four urban and four rural dis- tricts. Schools were selected using a two-stage stratified random sam- pling process. In the first stage they were stratified by geographical location (urban or rural), and subsequently by socio-economic status (low or middle). Private schools and schools inaccessible by roads (including farm schools) were excluded. The sampling protocol em- ployed a 95\% confidence level and a $3 \%$ margin of error. The sampling frame 
included 646 primary schools, and the final sample included 29 schools from low and middle income communities. Two classes each from grade 2, 4, and 6 were randomly selected to participate in the study. The original English questionnaire was translated to Afrikaans and isiXhosa using the backward translation method. The children could select their language preference in which to complete the ques- tionnaire from amongst the different language versions. The total sample included 3407 children between 8 and 12 years of age (8-year olds, $n=1032 ; 10$-year olds, $n=1109 ; 12$-year olds, $n=1143$ ).

\subsection{Instrumentation}

\subsubsection{Students' Life Satisfaction Scale (Huebner, 1991)}

The 7-item Students' Life Satisfaction Scale (SLSS) was developed to assess children's (ages 8-18 years) global life satisfaction (Huebner, 1991). The items are context-free and require respondents to evaluate their satisfaction on a 5-point Likert scale ranging from "very much disagree" (0), to "very much agree" (4). In the study it had been rescaled to a $0-10$ end-labelled scale with verbal anchors of "strongly disagree" (0) to "strongly agree" (10). The initial version of the scale comprised 10 items and was later reduced to 7-items owing to further item ana- lysis, as well as data and reliability estimates (Huebner, Suldo, \& Valois, 2003). For Wave 2 of the Children's Worlds survey, a modified 5-item SLSS was employed, including the following items: "My life is going well", "My life is just right", "I have a good life", and "I have what I want in life"; with the fifth item adapted from Diener, Emmons, Larsen, and Griffin's (1985) Satisfaction With Life Scale (SWLS), namely "The things in my life are excellent” (Rees \& Main, 2015).

The scale has been shown to display acceptable internal consistency, with alpha coefficients of 0.82 (Huebner, 1991; Huebner et al., 2004), 0.86 (Dew \& Huebner, 1994), and 0.89 (Marques et al., 2007). The SLSS has also evinced convergent validity by correlating well with other life satisfaction measures (Dew \& Huebner, 1994; Huebner, 1991) and overall life satisfaction (Casas, Bello, et al., 2013). The scale has been shown to display good criterion (Huebner et al., 2003), discriminant (Huebner \& Alderman, 1993), and predictive validity (Suldo \& Huebner, 2004). In the current study the SLSS has been transformed into a 100point scale for the purpose of comparison between scales.

\subsubsection{Personal Well-Being Index-School Children (Cummins \& Lau, 2005)}

The Personal Well-Being Index-School Children (PWI-SC) was de- veloped by Cummins and Lau (2005) to assess children's SWB, and is based on the adult version of the scale by Cummins, Eckersley, Pallant, van Vugt, and Misajon (2003). The scale evaluates a number of life satisfaction domains, such as standard of living, health, achieving in life, personal relationships, safety, community-connectedness, and fu- ture security (Cummins \& Lau, 2005). This 7-item scale, which is the- oretically entrenched, fundamentally endeavours to expound the global question of "How satisfied are you with your life as a whole?" (Cummins \& Lau, 2005). The original 7-item scale is often adapted to include items on religion/spirituality and school experience. The item on school experience has been included in the current study. Response options for the PWI-SC uses an 11-point rating scale ranging from "complete dissatisfaction" (o), and "complete satisfaction" (10). While 
the PWI was designed for the general adult population (PWI-A), the term "satisfaction" in the PWI-A, was replaced with "happiness" in the PWI-SC, and has been shown to yield similar results (Cummins et al., 2001, as cited in Cummins \& Lau, 2005; Lau, Cummins, \& McPherson, 2005). The PWI-SC has shown acceptable levels of reliability ( $\alpha=0.83$ in Casas \& Rees, 2015; $\alpha=0.82$ in Tomyn \& Cummins, 2011; Casas et al., 2014). In the current study, the PWI-SC has been transformed into a 100-point scale for the purpose of comparison between scales.

\subsubsection{Single Item on Overall Life Satisfaction}

A single- item measure assessing Overall Life Satisfaction (OLS) was included, on an endlabelled $0-10$ scale, (from "Not at all sa- tisfied" $=0$, to "Completely satisfied" $=10$ ) using the phrasing as sti- pulated by Cummins and Lau (2005): "How satisfied are you with your life as a whole?" The importance of including a single- item on life satisfaction was identified by Campbell et al. (1976) and further cor- roborated by Cummins and Lau (2005). Recent research has also found high reliability estimates (Lucas \& Donnellan, 2012) and criterion va- lidity (Cheung \& Lucas, 2014), whilst others (Casas \& Rees, 2015) note the use of the OLS as a means to ascertain convergent validity of SWB scales.

\subsection{Procedure and ethics}

The participation of each country in the project was dependent on obtaining ethics clearance from the institutions at which the principal investigators were based (see Rees \& Main, 2015). For the Children's Worlds Project, a broad set of ethics guidelines were devised, and highlighted the significance of: "(a) children having an active and in- formed choice in whether to participate in the survey or not; (b) ap- propriate measures to safeguard children's privacy, confidentiality and anonymity; and (c) a commitment to feeding back findings to partici- pating schools." (Rees \& Main, 2015, p.17). Informed parental consent differed in each country. Some countries employed either active or passive consent, while in others only the consent from the school was required. Regardless of the parental consent process, only children who provided active consent participated in the study.

The questionnaire was administered either by members of the re- search team, or a trained staff member at the schools in each country. Data were then captured and cleaned by the research team in the participating country, and sent to the international project manager for further scrutiny and weighting. In the current study, the unweighted datasets were used.

Preparation and depuration of the datasets for the current study followed two steps. In the first, the datasets of the three countries were combined into a master dataset. In the second step a missing data analysis was conducted with the SLSS and PWI-SC. Cases with more than three missing cases were deleted and those with two or less were substituted by regression. 


\subsection{Data analysis}

The study used Confirmatory Factor Analysis (CFA) and Structural Equation Modelling (SEM) using Maximum Likelihood Estimation in AMOS (version 24) to analyse the data. Structural Equation Modelling is a general set of data analysis techniques wherein specified theoretical models, indicating the relationship between observed and unobserved variables, are assessed against a set of observed data. The estimation of parameters and assessment of model fit are the key aims of SEM (Hox \& Bechger, 1998). If a good-fitting model exists, the researcher is able to validly assess the strength and nature of causal paths between variables. It is recommended that more than one fit index be used to assess model fit (Hooper, Coughlan, \& Mullen, 2008). Following re- commendations by Jackson, Gillaspy, and Purc-Stephenson (2009) and Kline (2010), the Comparative Fit Index (CFI), Root Mean Square Error of Approximation (RMSEA) and the Standardised Root Mean Square Residual (SRMR) were used as fit indexes in the current study. These recommendations have been used in a number of studies on children's well-being by Casas (see e.g. Casas et al., 2012; Casas et al., 2014) using cut-scores of $>0.980$ accepted for CFI and scores $<0.05$ regarded as a good fit for RMSEA and SRMR.

To facilitate the comparison of the results between the three coun- tries, measurement invariance was tested by means of multi-group confirmatory factor analysis (MGCFA). Measurement invariance refers to the extent to which items on a scale have the same meaning across groups and is a pre-requisite for meaningful comparison across groups (Meredith, 1993). If invariance is not tenable, then group differences in means or regression coefficients on the measured variables can be ac- counted for by true differences in group distributions or to a different understanding of the items (Casas et al., 2012), which ultimately results in unreliable or ambiguous interpretations (Millsap \& Olivera-Aguilar, 2012).

Measurement invariance is generally assessed on a hierarchical structure wherein incrementally restrictive constraints are applied to nested models. Measurement invariance is tenable if the model fit does not worsen by more than 0.010 on the CFI (Cheung \& Rensvold) and by 0.015 on the RMSEA and SRMR (Chen, 2007). In the current study, invariance of multi-group (country) models were tested in three steps.

In the first step, configural factor invariance, which assesses an un- constrained multigroup model wherein the parameters are freely es- timated, was tested. This unconstrained model represents the baseline against which further nested models are tested. Thereafter, metric factor invariance, which is a requisite for comparing covariance, correlations or regression coefficients, was tested by constraining the factor loadings of the baseline model. Finally, scalar factor invariance, which is a ne- cessary condition for comparing means between groups, was tested by constraining the factor loadings and intercepts. To test convergent va- lidity, a Structural Equation Model was tested by including the single- item OLS into the overall model. 
However, noting the contention of Milfont and Fischer (2010) that full scalar invariance is unlikely to hold in practice, partial measure- ment invariance is often considered. The concept of partial measure- ment invariance was introduced by Byrne, Shavelson, and Muthen (1989) and refers to the practice wherein a subset of the parameters is constrained to be invariant, while other parameters are allowed to be freely estimated. This allows for meaningful cross-group comparisons in the cases where full invariance is not obtained (Milfont \& Fischer, 2010). In the current study partial measurement invariance was applied in an exploratory manner, by the trial and error assessment of a range of nested models wherein various combinations of parameters were relaxed to obtain the best fit.

\section{Results}

Skewness of the items ranged from -1.340 to -1.951 for the SLSS and -1.184 to 2.425 for the PWI-SC, with Kurtosis from 0.918 to 3.532 for the SLSS and 0.212 to 6.168 for the PWI-SC. These departures from normality were attended to using the Bootstrap method (500 re- samples) as specified in AMOS (version 24). Cronbach Alpha for the SLSS was an acceptable 0.872 and 0.672 for the PWI-SC. Tables 1-2 show the mean scores of the items of the three scales across countries, whilst Table 3 depicts the mean composite scores of the SLSS and PWI- SC (transformed into 100 point scales). High scores were obtained for all countries, with Algeria scoring higher on both scales.

\subsection{Confirmatory Factor Analysis}

In order to assess the validity of the factorial structure of the scales, Confirmatory Factor Analysis was used (Maximum Likelihood Estimation) to test the fit statistics of various models (presented in Table 4). Initial models for the SLSS and PWI-SC showed adequate fit (Model 1 and Model 6). However, a modified model with one error co- variance (item 4 to item 5) for the SLSS (Model 2 in Table 4); and two co-variances (item 1 to item 4; item 6 to item 7) for the PWI-SC (Model 7 in Table 4) produced excellent fit (see Figs. 1 and 2).

\subsection{Multi-group confirmatory factor analysis}

Measurement invariance was assessed in three sequential steps wherein incrementally restrictive constraints were applied. In the first step an unconstrained multi-group model (configural invariance) was tested and formed the baseline model against which other models were tested. In the current study, for both the SLSS and the PWI-SC adequate fit statistics were found for the unconstrained multi-group models (Model $3 \& 8$ in Table 4). As the baseline models showed adequate fit, metric factor invariance was tested by constraining the factor loadings of the two unconstrained models. Given that the fit statistics did not worsen by more than 0.010 on the CFI (Cheung \& Rensvold, 2002) and 0.015 on the RMSEA and SRMR (Chen, 2007), metric invariance was found to be tenable for both latent variables (Model 4 for the SLSS \& Model 9 for the PWI-SC). Finally, scalar factor invariance was tested by constraining the measurement loadings and intercepts. Scalar factor invariance was found to be tenable for the SLSS (Model 5 in Table 4) but not for the PWI-SC (Model 10 in Table 4). 
In the current study partial measurement invariance for the PWI-SC was considered in an exploratory manner, by the trial and error as- sessment of a range of nested models wherein various combinations of parameters were relaxed to obtain the best fit. The best fitting model for the PWI-SC (Model 11 in Table 4) which met the requirements for scalar invariance allowed for items 1, 6 and 7 to be freely estimated, while four items (items 2-5) were held invariant (Table 5). The applicable items which can be compared across correlations, regressions and means are: satisfied with your health, satisfied with things that you are good at, satisfied with your relationships in general and satisfied with your safety (see Table 6 for the standardised regression weights).

\section{Table 1}

SLSS mean scores across the three countries.

\begin{tabular}{|c|c|c|c|c|c|c|c|c|}
\hline \multicolumn{9}{|l|}{ Country of survey } \\
\hline & \multicolumn{2}{|c|}{ Algeria } & \multicolumn{2}{|c|}{ Ethiopia } & \multicolumn{2}{|c|}{ South Africa } & \multicolumn{2}{|l|}{ Total } \\
\hline & Mean & SD & Mean & SD & Mean & SD & Mean & SD \\
\hline $\begin{array}{c}\text { My life is going } \\
\text { well }\end{array}$ & 8.93 & 1.974 & 8.32 & 2.261 & 8.49 & 2.460 & 8.61 & 2.242 \\
\hline My life is just right & 8.73 & 2.079 & 7.99 & 2.405 & 8.37 & 2.519 & 8.40 & 2.347 \\
\hline I have a good life & 8.91 & 2.081 & 7.95 & 2.506 & 8.39 & 2.591 & 8.46 & 2.416 \\
\hline $\begin{array}{l}\text { I have what I want } \\
\text { in life }\end{array}$ & 8.07 & 2.705 & 7.58 & 2.723 & 7.61 & 3.062 & 7.77 & 2.843 \\
\hline $\begin{array}{l}\text { The things in my } \\
\text { life are } \\
\text { excellent }\end{array}$ & 8.66 & 2.288 & 8.07 & 2.420 & 7.79 & 2.937 & 8.20 & 2.585 \\
\hline
\end{tabular}


Table 2

PWI-SC mean scores across the three countries.

\begin{tabular}{|c|c|c|c|c|c|c|c|c|}
\hline Country of survey & & & & & & & & \\
\hline \multirow[b]{2}{*}{ Satisfied with: } & \multicolumn{2}{|c|}{ Algeria } & \multicolumn{2}{|c|}{ Ethiopia } & \multicolumn{2}{|c|}{ South Africa } & \multicolumn{2}{|l|}{ Total } \\
\hline & Mean & SD & Mean & SD & Mean & SD & Mean & SD \\
\hline $\begin{array}{c}\text { All the things you } \\
\text { have }\end{array}$ & 7.97 & 2.665 & 7.22 & 3.099 & 8.79 & 2.068 & 8.02 & 2.696 \\
\hline Your health & 9.16 & 1.794 & 8.84 & 1.951 & 8.83 & 2.119 & 8.96 & 1.959 \\
\hline $\begin{array}{l}\text { The things you } \\
\text { want to be } \\
\text { good at }\end{array}$ & 9.08 & 1.742 & 8.74 & 1.820 & 8.80 & 2.072 & 8.89 & 1.885 \\
\hline How safe you feel & 9.00 & 1.843 & 8.18 & 2.252 & 8.34 & 2.504 & 8.54 & 2.228 \\
\hline $\begin{array}{l}\text { Your relationships } \\
\text { with people in } \\
\text { general }\end{array}$ & 8.88 & 1.919 & 8.41 & 2.095 & 8.16 & 2.642 & 8.51 & 2.254 \\
\hline $\begin{array}{l}\text { Doing things away } \\
\text { from your } \\
\text { home }\end{array}$ & 7.30 & 3.380 & 7.55 & 2.721 & 7.19 & 3.373 & 7.34 & 3.204 \\
\hline $\begin{array}{l}\text { What may happen } \\
\text { to you later in } \\
\text { your life }\end{array}$ & 8.68 & 2.239 & 7.55 & 2.724 & 7.88 & 2.956 & 8.09 & 2.679 \\
\hline
\end{tabular}

Table 3

Composite mean scores for the SLSS and PWI-SC across the three countries (100 point scale).

\begin{tabular}{|c|c|c|c|c|c|c|c|c|}
\hline & \multicolumn{2}{|c|}{ Algeria } & \multicolumn{2}{|c|}{ Ethiopia } & \multicolumn{2}{|c|}{ South Africa } & \multicolumn{2}{|c|}{ Total } \\
\hline & Mean & SD & Mean & SD & Mean & SD & Mean & SD \\
\hline SLSS & 86.60 & 18.51 & 79.79 & 20.39 & 81.30 & 21.44 & 82.87 & 20.28 \\
\hline PWI-SC & 85.83 & 13.49 & 80.70 & 13.63 & 82.84 & 15.09 & 83.35 & 14.24 \\
\hline OLS & 90.40 & 19.72 & 86.80 & 19.37 & 86.00 & 24.09 & 87.90 & 21.27 \\
\hline
\end{tabular}


Table 4

Fit indexes for the overall pooled data, multi-group \& SEM.

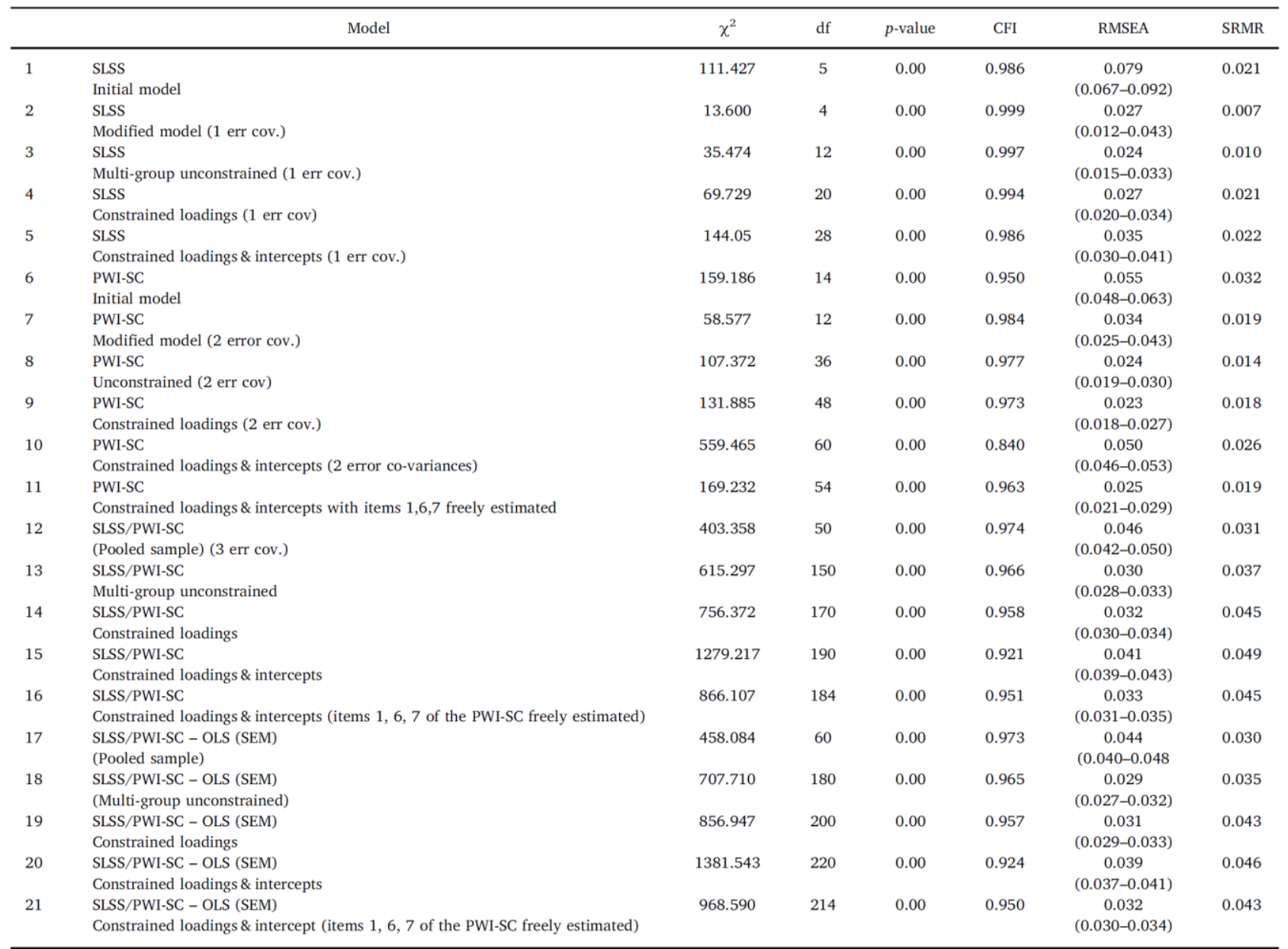




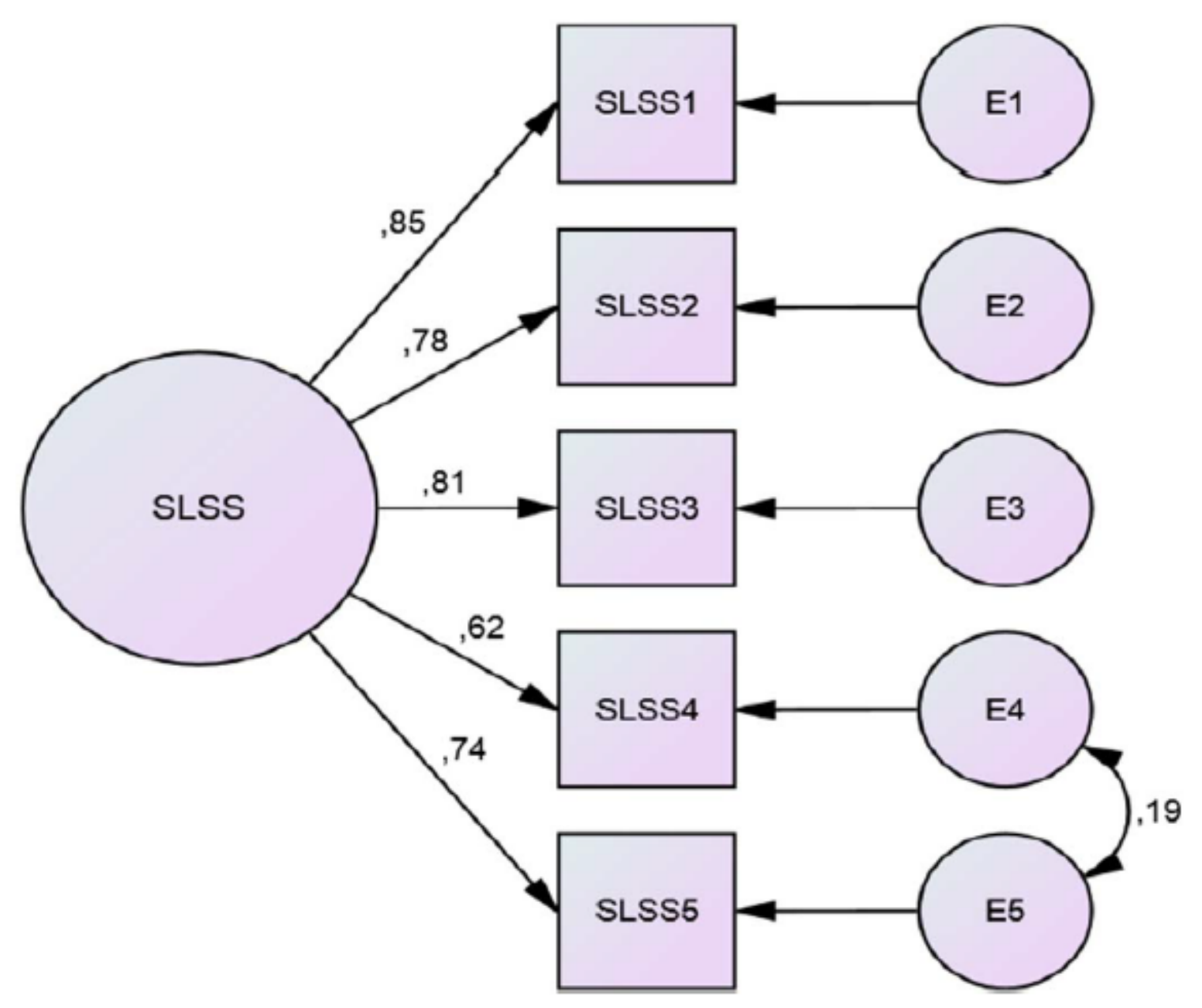

Fig. 1. Overall model SLSS.

\subsection{Structural Equation Modelling}

After assessing the fit structure of the multi-group models of the individual measures, a combined model including both the SLSS and the PWI-SC was tested. This strategy for testing a combination of dif- ferent scales measuring context-free and domain-specific life satisfac- tion is recommended by Casas (2016) who notes its usefulness for as- sessing cross-cultural, cross-national, and cross-linguistic comparability. Casas et al. (2012) and Casas, et al. (2014) in fact found that models including both context-free and domainspecific measures, with different levels of abstraction, showed improved model fit. This is in line with a seminal article by Stones and Kozma (1985) who report a hierarchical model of Happiness/SWB scales, showing a single second- order factor. In the current study the fit structure for the combined model is presented in Table 4 (Model 12-15), where appropriate fit was obtained for a modified model with three error co-variances (Model 12 in Table 4). Furthermore, when testing for measurement invariance across the three countries, configural and metric invariance was found to be tenable (Models 13-14 in Table 4), while the criteria for scalar invariance was not met (Model 15 in Table 4). These findings suggest that the combined model is comparable across the three countries by correlations and regressions, but that mean scores are not comparable. However, after the application of partial constraints on items 1, 6 and 7 of the PWI-SC, an adequate fit was obtained (Model 
16 in Table 4). In Models 17 measures was tested in a SEM model by regressing the latent variables onto the observed variable Overall Life Satisfaction (OLS) within the combined model.

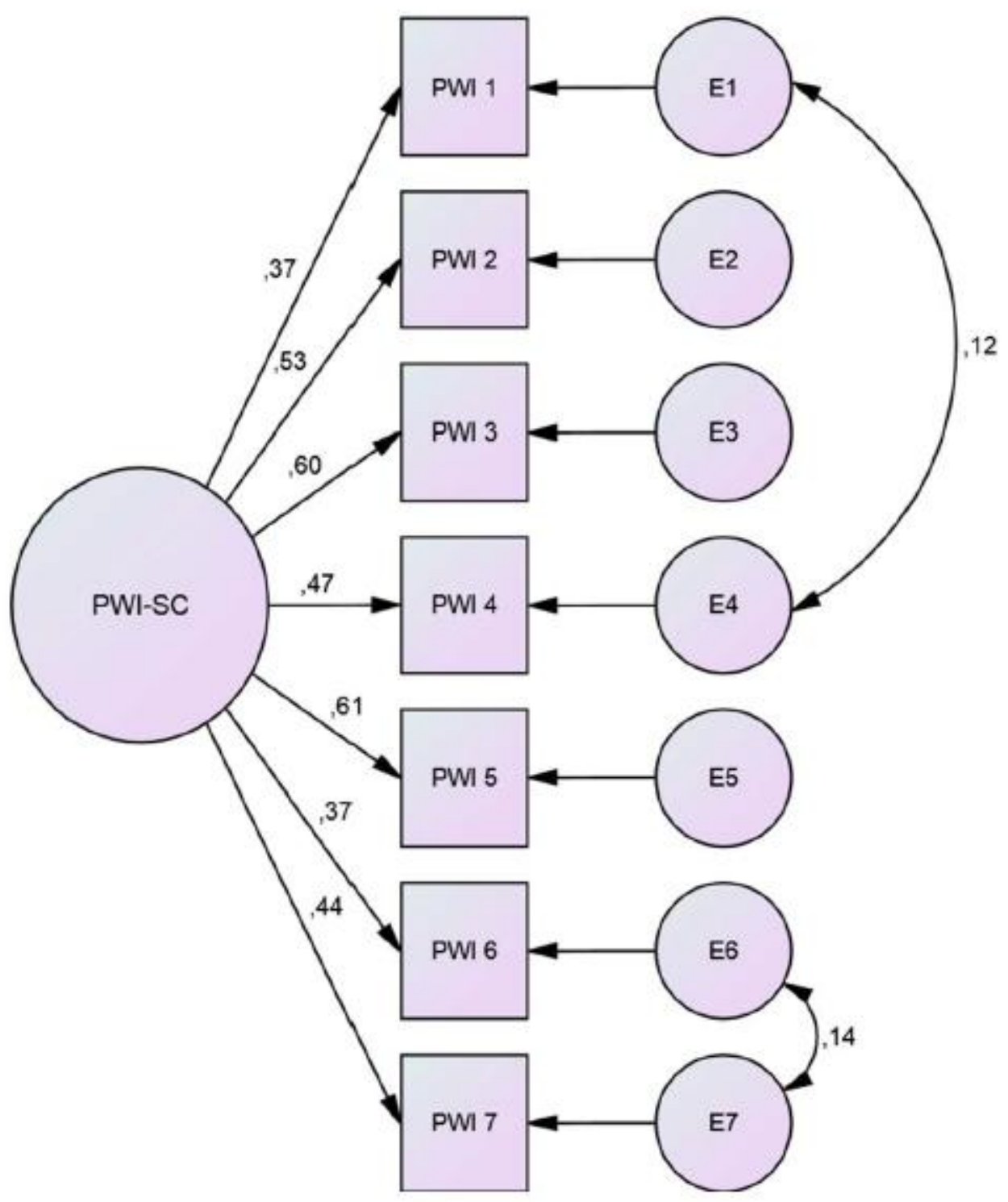

Fig. 2. Overall model PWI-SC.

Adequate fit structure, and high factor loadings, was obtained for the overall model with pooled data (Model 17 in Table 4) and the multi-group unconstrained (Model 18 in Table 4), confirming evidence of convergent validity. Thereafter, measurement invariance was tested, with metric invariance found to be tenable (Model 19 in Table 4), but not scalar invariance (Model 20 in Table 4). The appli- cation of partial constraints, with items 1, 6, and 7 being freely esti- mated resulted in scalar invariance being obtained (Model 21 in Table 4). Fig. 3 shows the standardised estimates of the overall model (including the OLS) and Table 6 presents the specific standardised re- gression weights (with partial constrained loadings and intercepts). Standardised estimates show adequate loadings for all 
items across the three countries. The standardised regression weights also show adequate loadings of the latent variables onto the OLS for the overall model, and across the three countries.

\section{Discussion}

The study aimed to determine children's SWB in three African countries. The study further aimed to assess the extent to which con- text-free (SLSS) and domain-specific (PWI-SC) scales are comparable across the three countries.

Mean composite scores for the scales showed high scores across all three countries, with Algeria presenting with higher scores than Ethiopia and South Africa. These high mean scores are comparable to those obtained in samples from developed countries (Casas et al., 2014) and higher than the mean range of 70-80 for child samples identified using Cummin's homeostatic theory (see Casas et al., 2008; Tomyn \& Cummins, 2011; Cummins, 1997; Marriage \& Cummins, 2004; Cummins, 2014; Savahl et al., 2016). These high scores, while typical of the negative skew present in SWB data, should be cautiously inter- preted as they are incongruent to the objective child well-being in- dicators of the countries wherein the children reside. These high scores (negative skew) is explained by Cummins (1995) who points to a number of studies which make reference to a life optimism bias', where individuals evaluate and put forward a 'generalised positive self-view' (Cummins et al., 2003). This positive evaluation of life experiences is sometimes portrayed regardless of unsatisfactory social contexts and living conditions; Zapf (1984, as cited in Olsen \& Schober, 1993) refers to this as the 'satisfaction paradox'. This notion of the 'satisfaction paradox' was forthcoming in the results of the current study as the participants presented with positive life views within the context of adverse social circumstances. From a homeostasis theory perspective, the results suggest that SWB is maintained at a high 'setpoint' by a range of internal and external buffers (Cummins, 2014).

Confirmatory factor analysis showed appropriate fit statistics for the pooled sample for both the context-free (SLSS) and domain-specific (PWI-SC) scales. Using MGCFA, the study found that the measures are comparable across the three countries. For the SLSS, the tenability of scalar factor invariance points to meaningful comparison across the three countries by correlations, regressions and means. For the PWI-SC, partial scalar invariance was achieved with four items (satisfied with your health, satisfied with things you want to be good at, satisfied with your relationships in general, and satisfied with your safety), allowing for comparisons by correlations, regressions, and means of the four items. Algeria presented with higher factor loadings for all the items on the SLSS and the PWI-SC, with the exception of the item the "things in life are excellent" higher mean scores than Ethiopia and South Africa on both measures. 
Table 5

Standardised regression weights for items on the SLSS (constrained loadings \& intercepts) and the PWI-SC (constrained loadings \& intercepts of items 2-5).

\begin{tabular}{|c|c|c|c|c|c|c|c|c|c|c|}
\hline \multirow{2}{*}{$\begin{array}{l}\text { Bootstrap ML, } 95 \text { CI } \\
\text { Resamples }=500\end{array}$} & & \multicolumn{3}{|l|}{ Algeria } & \multicolumn{3}{|l|}{ Ethiopia } & \multicolumn{3}{|c|}{ South Africa } \\
\hline & & Estimate & Lower & Upper & Estimate & Lower & Upper & Estimate & Lower & Upper \\
\hline \multicolumn{11}{|l|}{ SLSS } \\
\hline LifeGoingWell (SLSS1) & $\leftarrow$ SLSS & 0.862 & 0.825 & 0.894 & 0.840 & 0.807 & 0.869 & 0.829 & 0.790 & 0.869 \\
\hline LifeJustRight (SLSS2) & $\leftarrow$ SLSS & 0.777 & 0.721 & 0.821 & 0.775 & 0.725 & 0.814 & 0.770 & 0.720 & 0.817 \\
\hline HaveGoodLife (SLSS3) & $\leftarrow$ SLSS & 0.831 & 0.787 & 0.873 & 0.818 & 0.774 & 0.858 & 0.793 & 0.749 & 0.839 \\
\hline HaveWhatIWantInLife (SLSS4) & $\leftarrow$ SLSS & 0.619 & 0.569 & 0.699 & 0.666 & 0.610 & 0.710 & 0.599 & 0.552 & 0.645 \\
\hline ThingsLifeExcellent (SLSS5) & $\leftarrow$ SLSS & 0.752 & 0.699 & 0.800 & 0.778 & 0.819 & 0.819 & 0.682 & 0.639 & 0.732 \\
\hline "Mean differences between countries & & & & & $b-0.711$ & -0.859 & -0.541 & b -0.497 & -0.648 & -0.328 \\
\hline \multicolumn{11}{|l|}{ PWI-SC } \\
\hline Health (PWI-SC2) & $\leftarrow$ PWI-SC & 0.560 & 0.493 & 0.622 & 0.478 & 0.419 & 0.542 & 0.521 & 0.454 & 0.588 \\
\hline ThingsGoodAt (PWI-SC3) & $\leftarrow$ PWI-SC & 0.625 & 0.556 & 0.685 & 0.539 & 0.489 & 0.594 & 0.578 & 0.508 & 0.644 \\
\hline Relationships (PWI-SC4) & $\leftarrow$ PWI-SC & 0.529 & 0.465 & 0.593 & 0.457 & 0.406 & 0.512 & 0.421 & 0.365 & 0.477 \\
\hline Safety (PWI-SC5) & $\leftarrow$ PWI-SC & 0.739 & 0.679 & 0.794 & 0.551 & 0.497 & 0.604 & 0.585 & 0.523 & 0.643 \\
\hline "Mean differences between countries & & & & & ${ }^{b}-0.426$ & -0.553 & -0.304 & ${ }^{b}-0.409$ & -0.537 & -0.292 \\
\hline
\end{tabular}

a Algeria is used as the point of reference.

${ }^{\mathrm{b}}$ Indicates significant mean differences.

Table 6

Standardised regression weights (SEM with OLS) for items on the SLSS (constrained loadings \& intercepts) and the PWI-SC (constrained loadings \& intercepts of items 2-5)

\begin{tabular}{|c|c|c|c|c|c|c|c|c|c|c|}
\hline \multirow{2}{*}{$\begin{array}{l}\text { Bootstrap ML, } 95 \text { CI } \\
\text { Resamples }=500\end{array}$} & & \multicolumn{3}{|l|}{ Algeria } & \multicolumn{3}{|l|}{ Ethiopia } & \multicolumn{3}{|c|}{ South Africa } \\
\hline & & Estimate & Lower & Upper & Estimate & Lower & Upper & Estimate & Lower & Upper \\
\hline \multicolumn{11}{|l|}{ SLSS: } \\
\hline OLS & $\leftarrow$ SLSS & 0.616 & 0.539 & 0.677 & 0.549 & 0.467 & 0.626 & 0.473 & 0.390 & 0.552 \\
\hline LifeGoingWell & $\leftarrow$ SLSS & 0.863 & 0.830 & 0.894 & 0.842 & 0.811 & 0.872 & 0.838 & 0.801 & 0.874 \\
\hline LifeJustRight & $\leftarrow$ SLSS & 0.775 & 0.721 & 0.817 & 0.773 & 0.724 & 0.812 & 0.770 & 0.722 & 0.815 \\
\hline HaveGoodLife & $\leftarrow$ SLSS & 0.818 & 0.776 & 0.860 & 0.812 & 0.766 & 0.852 & 0.778 & 0.736 & 0.820 \\
\hline ThingsLifeExcellent & $\leftarrow$ SLSS & 0.764 & 0.714 & 0.811 & 0.779 & 0.740 & 0.816 & 0.686 & 0.642 & 0.732 \\
\hline \multicolumn{11}{|l|}{ PWI-SC: } \\
\hline OLS & $\leftarrow$ PWI-SC 0.623 & 0.540 & 0.699 & 0.649 & 0.565 & 0.717 & 0.583 & 0.484 & 0.670 & \\
\hline SatisfiedHealth & $\leftarrow$ PWI-SC & 0.581 & 0.521 & 0.643 & 0.502 & 0.447 & 0.554 & 0.536 & 0.471 & 0.594 \\
\hline SatisfiedThingsGoodAt & $\leftarrow$ PWI-SC & 0.598 & 0.529 & 0.658 & 0.509 & 0.458 & 0.558 & 0.538 & 0.467 & 0.609 \\
\hline SatisfiedRelationships & $\leftarrow$ PWI-SC & 0.540 & 0.484 & 0.602 & 0.475 & 0.425 & 0.526 & 0.417 & 0.366 & 0.467 \\
\hline SatisfiedSafety & $\leftarrow$ PWI-SC & 0.721 & 0.669 & 0.771 & 0.550 & 0.499 & 0.603 & 0.567 & 0.503 & 0.627 \\
\hline
\end{tabular}




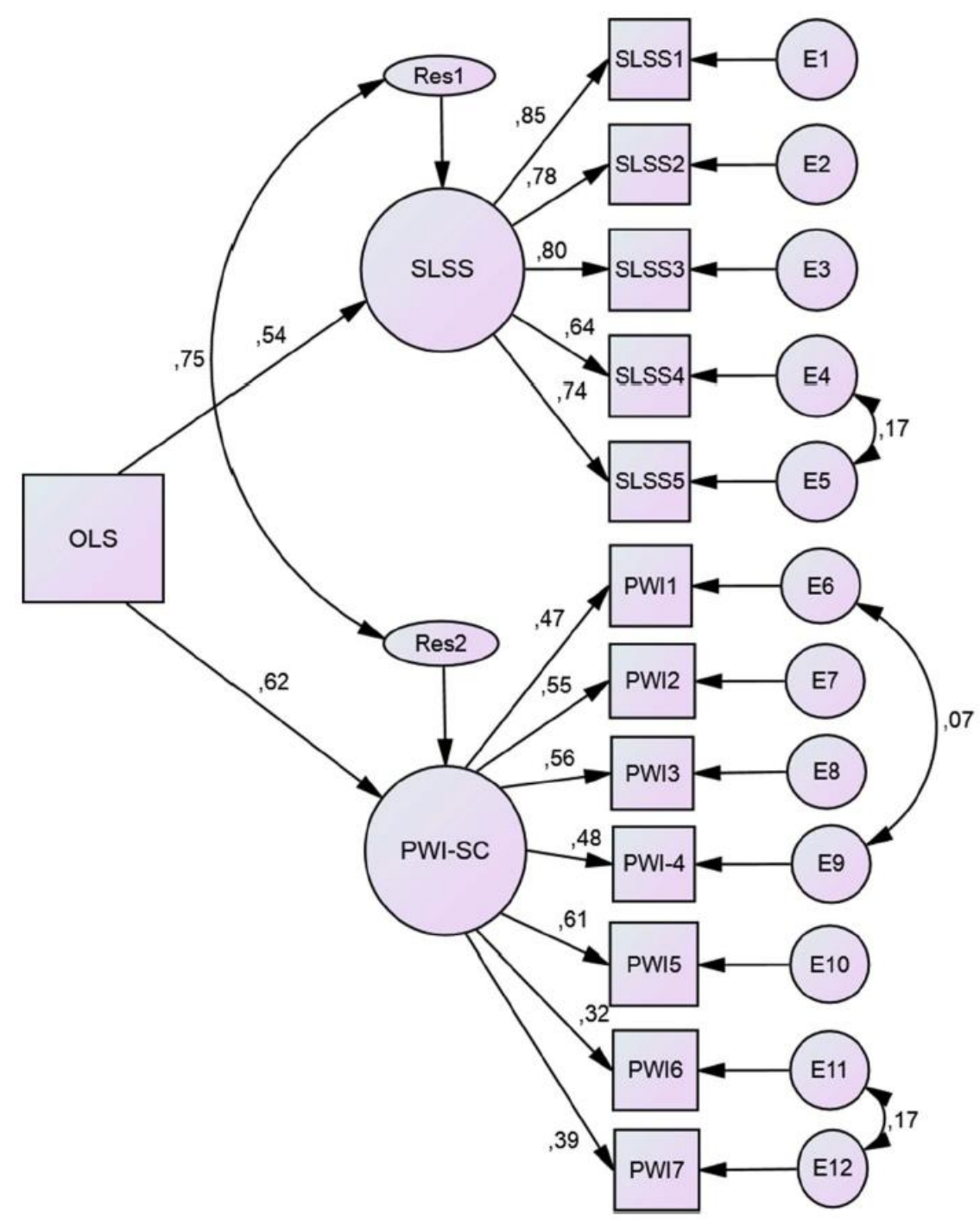

Fig. 3. Structural Equation Model (with pooled data).

Appropriate fit structure was obtained for the combined model and the SEM including the single-item scale of OLS. This finding lends support to the contention of Casas (2016) who has advised on the usefulness of including multiple scales testing various levels of abstraction when investigating SWB. Convergent validity of the scales was established as adequate factor loadings were obtained for the latent variables when regressed onto the OLS.

Two tentative conclusions are therefore forthcoming. Firstly, that the SLSS and PWI-SC are valid measures to use in the three African countries and that meaningful comparisons can be made across the three countries; and secondly, that the Algerian sample scores sig- nificantly higher on SWB than Ethiopia and South Africa, measured using both context-free and domain-specific scales.

The fact that the MGCFA demonstrated that the measures are comparable across the three countries implies that cultural variation, response bias, and response styles are not substantially influencing the scores. The key question then arises: what factors are responsible for the significant differences in scores between the three countries? Intuitively, 
one would suspect that the objective indicators of the countries are playing a central role. A cursory look at the objective indicators, suggest that Algeria performs better than South Africa and Ethiopia on the key indicators, with the exception of GDP. However, it is wellestablished in the literature that a country's GDP does not ac- curately reflect the well-being of its citizenry. Recently Diener, Tay, and Oishi (2013) have speculated that increases in SWB are likely to occur when rising income is accompanied by increases in material welfare, satisfaction with finances, and optimism about the future; while others such as Roberts and DelVecchio (2000) found that psychological factors (such as personality), are less stable in explaining SWB (both in adults and children), and that SWB is more reactive to contextual factors in childhood. The next critical question is: will concomitant increases in adults' material welfare trickle down to eventually make significant improvements in children's SWB? There is some evidence in the lit- erature, where Main (2014) found that material deprivation to be a significant predictor of children's SWB. Contexts characterised by ma- terial deprivation and poverty can therefore be intuitively associated with lower SWB. The logical conclusion is that increasing children's access to material resources and reducing the effects of poverty would lead to increases in their SWB, at least until a certain level. While this may be a plausible course of action in certain contexts, in developing economies such as Africa, it is more likely that a combination of fore- grounding psychological strengths such as hope (Savahl, Isaacs, Adams, Carels, \& September, 2013), decreasing material deprivation through appropriate government expenditure, and the advancement of policy initiatives aimed at improving children's position in society, would contribute to an increase in children's SWB. The current study con-tributes by validating measures to assess SWB with in-country samples of children, and to effect meaningful comparisons across the three countries.

The implication for policy is that governments need to take proac- tive measures to enhance the capacity of families to enable them to provide material and other basic needs of children, and mitigate pov- erty and deprivation which has a significant impact on the wellbeing of children. This in turn requires putting in place appropriate tax collec- tion and redistribution schemes targeting families in the lowest quin- tiles. Targeted social protection programmes are typical examples of such measures. As demonstrated in South Africa, such tailored social protection schemes have the potential to address deprivation and con- tribute to improved access to essential services by vulnerable children. The analysis in this study is based on only three countries, and it would be difficult to make generalisations about the rest of Africa. It is, therefore, recommended that further research be conducted with di- verse samples of children from other contexts in Africa. To this end, it is propounded that further cross-cultural and cross-linguistic testing of SWB measures be conducted, particularly in the Western regions of Africa. In-depth qualitative research may also shed more light on the psychological variables that could influence children's SWB.

Finally, many multi-country initiatives are currently being under- taken in Africa and illustrates a commitment to promote collaborative work towards achieving sustainable development in Africa. The area of child research should be prioritised in this direction. 
The New Partnership for Africa's Development (NEPAD) could potentially lead such efforts (http://www.nepad.org/).

\section{Acknowledgments}

The authors wish to thank the National Research Foundation of South Africa (project grant

number: 87931) and the Jacobs Foundation (grant number: 2015-1198) for financial and institutional support. 


\section{References}

Abubakar, Abubakar, A., van de Vijver, V., Alonso-Arbiol, I., He, J., Adams, B., Aldhafri, Tair, E., ... (2015). Measurement invariance of the brief multidimensional student's life satisfaction scale among adolescents and emerging adults across 23 cultural contexts. Journal of Psychoeducational Assessment, 1-11. http://dx.doi.org/10.1177/ 0734282915611284.

African Child Policy Forum (ACPF) (2013). The African report on child wellbeing 2013: Towards greater accountability to Africa's children. Addis Ababa: The African Child Policy Forum.

Alfaro, J., Guzman, J., García, C., Sirlopu, D., Reyes, F., \& Varela, J. (2016). Psychometric properties of the Spanish version of the Personal Wellbeing Index-School Children (PWI-SC) in Chilean school children. Child Indicators Research, 9, 731-742. http://dx. doi.org/10.1007/s12187-015-9342-2.

Alfaro, J., Guzman, J., Sirlopu, D., García, C., Reyes, F., \& Gaudlitz, L. (2016). Psychometric properties of Huebner's Satisfaction with Life in Students Scale (SLSS) in Chilean boys and girls between 10 and 12 years old. Annals of Psychology, $32(2)$, 383-392. http://dx.doi.org/10.6018/analesps.32.2.217441.

Allardt, E. (1976). Dimensions of welfare in a comparative Scandinavian study. Acta Sociologica, 19, 227-240.

Andrews, F. M., \& Withey, S. B. (1976). Social indicators of well-being. New York: Plenum. Ben-Arieh, A. (2010). Developing indicators for child well-being in a changing context. In C. McAuley, \& W. Rose (Eds.), Child well-being: Understanding children's lives (pp. 129- 142). London: Jessica Kingsley Publishers.

Byrne, B. M., Shavelson, R. J., \& Muthen, B. (1989). Testing for the equivalence of factor covariance and mean structures: The issue of partial measurement invariance. Psychological Bulletin, 105(3), 456-466.

Camfield, L., Streuli, N., \& Woodhead, M. (2010). Children's well-being in developing countries: A conceptual and methodological review. European Journal of Development Research, 22(3), 398-416.

Campbell, A., Converse, P. E., \& Rodgers, W. L. (1976). The quality of American life: Perceptions, evaluations and satisfaction. New York: Russell Sage.

Casas, Baltatescu, Bertran, Gonzalez, \& Hatos (2013). School satisfaction among adoles- cents: Testing different indicators for its measurement and its relationship with overall life satisfaction and subjective well-being in Romania and Spain. Social Indicators Research. http://dx.doi.org/10.1007/s11205-0120025-9.

Casas, F. (2016). Children, adolescents and quality of life: The social sciences perspective over two decades. In F. Maginno (Ed.), A life devoted to quality of life: Festschrift in honor of Alex C. Michalos (pp. 3-21). Dordrecht: Springer.

Casas, F., Bello, A., Gonzalez, M., \& Aligué, M. (2013). Children's subjective well-being measured using a composite index: What impacts Spanish first-year secondary 
edu- cation students' subjective well-being? Child Indicators Research. http://dx.doi.org/ 10.1007/s12187-013-9182-x.

Casas, F., Coenders, G., Cummins, R. A., González, M., Figuer, C., \& Malo, S. (2008). Does subjective well-being show a relationship between parents and their children? Journal of Happiness Studies, 9, 197-205.

Casas, F., \& Rees, G. (2015). Measures of children's subjective well-being: Analysis of the potential for cross-national comparisons. Child Indicators Research. http://dx.doi.org/ 10.1007/s12187-014-9293-z.

Casas, F., Sarriera, J. C., Abs, D., Coenders, G., Saforcada, J. A. E., \& Tonon, G. (2012). Subjective indicators of personal well-being among adolescents. Performance and results for different scales in Latin-language speaking countries: A contribution to the international debate. Child Indicators Research. Child Indicators Research, 5(1), 1-28.

Casas, F., Tiliouine, H., \& Figuer, C. (2014). The subjective well-being of adolescents from two different cultures: Applying three versions of the PWI in Algeria and Spain. Social Indicators Research. http://dx.doi.org/10.1007/s11205-0120229-z.

Chen, F. F. (2007). Sensitivity of goodness of fit indexes to lack of measurement invariance. Structural Equation Modeling: A Multidisciplinary Journal, 14(3), 464-504.

Cheung, F., \& Lucas, R. E. (2014). Assessing the validity of single-item life satisfaction measures: Results from three large samples. Quality of Life Research, 23(10), 2809-2818.

Cheung, G. W., \& Rensvold, R. B. (2002). Evaluating goodness-of-fit indexes for testing MI. Structural Equation Modelling, 9, 235-255.

Cummins, R. A. (1995). On the trail of the gold standard for subjective well-being. Social Indicators Research, 35, 179-200.

Cummins, R. A. (1997). The Comprehensive Quality of Life Scale (ComQol) (Fifth edition). Melbourne: School of Psychology, Deakin University.

Cummins, R. A. (2014). Understanding the well-being of children and adolescents through homeostatic theory. In A. Ben-Arieh, F. Casas, I. Frones, \& J. E. Korbin (Eds.), Handbook of child well-being: Theories, methods and policies in global perspective (pp. 635-661). Dordrecht: Springer.

Cummins, R. A., Eckersley, R., Pallant, J., van Vugt, J., \& Misajon, R. (2003). Developing a national index of subjective wellbeing: The Australian UNITY Wellbeing Index. Social Indicators Research, 64(2), 159-190.

Cummins, R. A., Eckersley, R., Pallant, J., Van Vugt, J., Shelley, J., Pusey, M., \& Misajon, R. (2001). Australian Unity Well-being Index: Report \#1. Australian Centre on Quality of Life: Deakin University (http://acqol.deakin.edu.au/index_wellbeing/index.html).

Cummins, R. A., \& Lau, A. D. L. (2005). Personal wellbeing index: School children (PWI-SC) (3rd ed.). Melbourne: Deakin University.

Dew, T., \& Huebner, E. S. (1994). Adolescents' perceived quality of life: An exploratory investigation. Journal of School Psychology, 33, 185-199. 
Diener, E., Emmons, R. A., Larsen, R. J., \& Griffin, S. (1985). The satisfaction with life scale. Journal of Personality Assessment, 49, 71-75.

Diener, E., Tay, L., \& Oishi, S. (2013). Rising income and the subjective well-being of nations. Journal of Personality and Social Psychology, 104, 267-276.

Habibi, M., Pooravari, M., Salehi, S., Fard, Z. G., \& Pooravari, M. (2015). Validity and reliability of the multidimensional student's life satisfaction scale among Iranian girl students. Journal of Research and Health, 5(1), 65-73.

Hooper, D., Coughlan, J., \& Mullen, M. (2008). Structural Equation Modelling: Guidelines for determining model fit. Electronic Journal of Business Research Methods, 6(1), 53-60.

Hox, J. J., \& Bechger, T. M. (1998). An introduction to structural equation modeling. Family Science Review, 11, 354-373.

Huebner, E. S. (1991). Initial development of the Student's Life Satisfaction Scale. School Psychology International, 12, 231-240.

Huebner, E. S. (1994). Preliminary development and validation of a multidimensional life satisfaction scale for children. Psychological Assessment, 6, 149-158.

Huebner, E. S., \& Alderman, G. L. (1993). Convergent and discriminant validation of a children's life satisfaction scale: Its relationship to self- and teacher-reported psy- chological problems and school functioning. Social Indicators Research, 30, $71-82$.

Huebner, E. S., Seligson, J. L., Valois, R. F., \& Suldo, S. M. (2006). A review of the Brief Multidimensional Students' Life Satisfaction Scale. Social Indicators Research, 79, 477-484.

Huebner, E. S., Suldo, S. M., \& Valois, R. F. (2003). Psychometric properties of two brief measures of children's life satisfaction: The students' life satisfaction scale (SLSS) and the Brief Multidimensional Students' Life Satisfaction Scale (BMSLSS). Paper prepared for the indicators of positive development conference, March 12-13 (Washington, DC).

Huebner, E. S., Valois, R. F., Suldo, S. M., Smith, L. C., McKnight, C. G., Seligson, J. L., \& Zullig, K. J. (2004). Perceived quality of life: A neglected component of adolescent health assessment and intervention. Journal of Adolescent Health, 34(4), 270-278.

Jackson, D. L., Gillaspy, J. A., Jr., \& Purc-Stephenson, R. (2009). Reporting practices in confirmatory factor analyses: An overview and some recommendations. Psychological Methods, 14(1), 6-23.

Jovanovic, V., \& Zuljevic, D. (2013). Psychometric evaluation of the Serbian version of the Multidimensional Students' Life Satisfaction Scale. Social Indicators Research, 110, 55-69.

Kline, R. B. (2010). Principles and practice of Structural Equation Modelling (3rd ed.). New York: Guilford Press.

Lau, A. L. D., Cummins, R. A., \& McPherson, W. (2005). An investigation into the cross- cultural equivalence of the Personal Wellbeing Index. Social Indicators Research, 72, 403-430. 
Lucas, R. E., \& Donnellan, B. M. (2012). Estimating the reliability of single-item life sa- tisfaction measures: Results from four national panel studies. Social Indicators Research, 105, 323. http://dx.doi.org/10.1007/s11205-011-9783-z.

Main, G. (2014). Child poverty and children's subjective well-being. Child Indicators Research, 7(3), 451-472.

Marques, S. C., Pais-Ribeiro, J. L., \& Lopez, S. J. (2007). Validation of a Portuguese version of the Students' Life Satisfaction Scale. Applied Research Quality Life, 2(83), http://dx.doi.org/10.1007/s11482-007-9031-5.

Marriage, K., \& Cummins, R. A. (2004). Subjective quality of life and self-esteem in children: The role of primary and secondary control in coping with everyday stress. Social Indicators Research, 66, 107-122.

Mekonen, Y., \& Dejene, N. (2015). Children's worlds national report: Ethiopia. The African Child Policy Forum.

Meredith, W. (1993). Measurement invariance, factor analysis, and factorial invariance. Psychometrika, 58, 525-542.

Milfont, T. L., \& Fischer, R. (2010). Testing measurement invariance across groups: Applications in cross-cultural research. International Journal of Psychological Research, 3(1), 111-121.

Millsap, R. E., \& Olivera-Aguilar, M. (2012). Investigating measurement invariance using confirmatory factor analysis. In R. H. Hoyle (Ed.), Handbook of structural equation modeling (pp. 380-392). New York: Guilford.

Olsen, G. I., \& Schober, B. I. (1993). The satisfied poor: Development of an intervention- oriented theoretical framework to explain satisfaction with a life in poverty. Social Indicators Research, 28(2), 173-193.

Rees, G., \& Main, G. (Eds.), (2015). Children's views on their lives and well-being in 15 countries: An initial report on the Children's Worlds survey, 2013-14. York, UK: Children's Worlds Project (ISCWeB).

Roberts, B. W., \& DelVecchio, W. F. (2000). The rank-order consistency of personality traits from childhood to old age: A quantitative review of longitudinal studies. Psychological Bulletin, 126(1), 3-25.

Sandin, B. (2014). History of children's well-being. In A. Ben-Arieh, F. Casas, I. Frones, \&

J. E. Korbin (Eds.), Handbook of child well-being: Theories, methods and policies in global perspective (pp. 31-86). Dordrecht: Springer.

Sarriera, J. C., Bedin, L., \& Abs, D. (2013). Testing different measures of Well-Being in Brazilian Children. Paper presented at the 4 th International Conference of the ISCI, 29- 3 May, 2013 (Seoul, Korea).

Sarriera, J. C., Casas, F., Alfaro, J., Bedina, L., Strelhow, M. R. W., Abs, D., ... Oyarzún, D. (2014). Psychometric properties of the Personal Wellbeing Index in Brazilian and Chilean adolescents including spirituality and religion. Psychology Psicologia Reflexão e Crítica, 27(4), 599-608. http://dx.doi.org/10.1590/1678-7153.201427411. 
Savahl, S., Adams, S., Isaacs, S., September, S., Hendricks, G., \& Noordien, Z. (2015). Subjective well-being amongst a sample of South African children: A descriptive study. Child Indicators Research, 8(1), 211-226.

Savahl, S., Casas, F., \& Adams, S. (2016). Children's subjective well-being: Multi-group analysis among a sample of children from two socio-economic status groups in the Western Cape, South Africa. Child Indicators Research. http://dx.doi.org/10.1007/ s12187-016-9392-0.

Savahl, S., Isaacs, S., Adams, S., Carels, C. Z., \& September, R. (2013). An exploration into the impact of exposure to community violence and hope on children's perceptions of well-being: A South African perspective. Child Indicators Research, 6(3), 579-592. http://dx.doi.org/10.1007/s12187-013-9183-9.

Siyez, D., \& Kaya, A. (2007). Validity and reliability of the Brief Multidimensional Students' Life Satisfaction Scale with Turkish children. Journal of Psychoeducational Assessment, 26(2), 139-147.

Stones, M. J., \& Kozma, A. (1985). Structural relationships among happiness scales: A second order factorial study. Social Indicators Research, 17, 19-28.

Suldo, S. M., \& Huebner, E. S. (2004). Does life satisfaction moderate the effects of stressful life events on psychopathological behaviour during adolescence? School Psychology Quarterly, 19(2), 93-105.

Tomyn, A. J., \& Cummins, R. A. (2011). The subjective wellbeing of high-school students: Validating the personal wellbeing index-school children. Social Indicators Research, 101, 405-418.

Tian, L., Zhang, J., \& Huebner, S. (2015). Preliminary validation of the brief multidimensional Students' life satisfaction scale (BMSLSS) among Chinese elementary school students. Child Indicators Research, 8, 907-923.

Tiliouine, H. (2012). Subjective wellbeing, psychological needs, meaning in life, religious practice and income in the population of Algeria. Journal of Social Research \& Policy, 2(3), 1-20.

Tiliouine, H., Cummins, R. A., \& Davern, M. (2006). Measuring wellbeing in developing countries: The case of Algeria. Social Indicators Research, 75, 1-30.

Tomyn, A. J., Norrish, J. M., \& Cummins, R. A. (2013). The subjective wellbeing of in- digenous Australian adolescents: Validating the personal wellbeing indexschool children. Social Indicators Research, 110, 1013-1031. http://dx.doi.org/10.1007/ s11205-011-9970-y.

Tomyn, A. J., Tamir, E., Stokes, M. A., \& Dias, P. C. (2015). A Cross-Cultural evaluation of the Personal Wellbeing Index-School Children in samples of Australian and Portuguese Adolescents. Applied Research in Quality of Life, 1-15. http://dx.doi.org/ 10.1007/s11482-015-9400-4.

Watts, H. W., \& Hernandez, D. J. (Eds.), (1982). Child and family indicators: A report with recommendations. Washington, DC: Center for Coordination of Research on Social Indicators.

Weber, M., Ruch, W., \& Huebner, E. S. (2013). Adaptation and initial validation of the German version of the Students' Life Satisfaction Scale (German SLSS). European Journal of Psychological Assessment, 29(2), 105-112. 
World Bank (2016). http://databank.worldbank.org/data/reports.aspx?source=worlddevelopment-indicators.

Ye, M., Li, L., Li, Y., Shen, R., Wen, S., \& Zhang, J. (2014). Life satisfaction of adolescents in Hunan, China: Reliability and validity of Chinese Brief Multidimens Students' Life Satisfaction Scale (BMSLSS). Social Indicators Research, 118, 515 